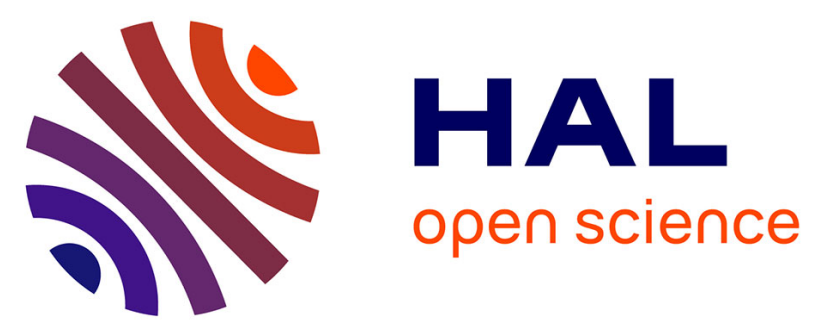

\title{
Growth and differentiation factor 11 (GDF11): Functions in the regulation of erythropoiesis and cardiac regeneration
}

Luc Rochette, Marianne Zeller, Yves Cottin, Catherine Vergely

\section{- To cite this version:}

Luc Rochette, Marianne Zeller, Yves Cottin, Catherine Vergely. Growth and differentiation factor 11 (GDF11): Functions in the regulation of erythropoiesis and cardiac regeneration. Pharmacology and Therapeutics, 2015, 156, pp.26-33. 10.1016/j.pharmthera.2015.10.006 . hal-03434120

\section{HAL Id: hal-03434120 \\ https://u-bourgogne.hal.science/hal-03434120}

Submitted on 18 Nov 2021

HAL is a multi-disciplinary open access archive for the deposit and dissemination of scientific research documents, whether they are published or not. The documents may come from teaching and research institutions in France or abroad, or from public or private research centers.
L'archive ouverte pluridisciplinaire HAL, est destinée au dépôt et à la diffusion de documents scientifiques de niveau recherche, publiés ou non, émanant des établissements d'enseignement et de recherche français ou étrangers, des laboratoires publics ou privés. 


\title{
Growth and differentiation factor 11 (GDF11): functions in the regulation of erythropoiesis and cardiac regeneration.
}

\author{
Luc ROCHETTE ${ }^{1}$, Marianne ZELLER ${ }^{1}$, Yves COTTIN ${ }^{1,2}$, and Catherine VERGELY ${ }^{1}$ \\ ${ }^{1}$ Laboratoire de Physiopathologie et Pharmacologies Cardio-Métaboliques (LPPCM) \\ ${ }^{2}$ Service de Cardiologie -CHU-Dijon
}

INSERM UMR866 - Université de Bourgogne - Facultés des Sciences de Santé.

7 Boulevard Jeanne d'Arc - 21033 Dijon Cedex - France

\section{ABSTRACT}

Members of the TGF- $\beta$ superfamily transduce their signals through type I and II receptor serine/threonine kinases. The binding of activins to activin type IIA (ActRIIA) or type IIB (ActRIIB) receptors induces the recruitment and phosphorylation of an activin type I receptor (ALK4 and/or ALK7), which then phosphorylates the Smad2 and Smad3 intracellular signaling proteins. The regulation of members of the TGF- $\beta$ family is known to be complex, because many proteins able to bind the ligands and inhibit their activities have been identified. Growth and differentiation factor 11 (Gdf11) belongs to the TGF- $\beta$ family. GDF11, like other members of the TGF- $\beta$ superfamily, is produced from precursor proteins by proteolytic processing. Recent studies have reported that GDF11-ActRIIB-Smad2/3-dependent signaling is a key regulatory mechanism in proliferating erythroid precursors as it controls their late-stage maturation. In mammalian tissues, aging is typically accompanied by a progressive loss of homeostasis and impaired regenerative potential. The administration of GDF11 is effective in experimental cardiac hypertrophy, and the identification of GDF11 as a "rejuvenating factor" opens up perspectives for the treatment of age-related cardiac dysfunction. Recent studies of the heart, skeletal muscle and central nervous system indicate that exposure to young blood reverses age-related impairments. The molecular mediators of this "rejuvenation" include growth factors such as GDF11 and cytokines such as CCL11. GDF11 and CCL11 are, perhaps, only the first two in a series of circulating molecules that will be found to influence the aging of different tissues; Are CCL11 and GDF11 endogenous factors for an "elixir of youth"?

Keywords: GDF11, CCL11, erythropoiesis, cardiac regeneration

Abbreviations: Act, activin; ActR, activin receptor type; ALK, activin receptor-like kinase; BMP, bone morphogenetic protein; BMPR, BMP receptor ; CCL11, chemokine (C-C motif) ligand 11; CHF, coronary heart failure; EPO, erythropoietin; Foxo 3, Forkhead box $\mathrm{O} 3$; GDF, growth and differentiation factor; GASP, GDF-associated serum protein; HO, heme-oxygenase; MI, myocardial infarction; NO, nitric oxide; NOS, nitric oxide synthase; Nox, NADPH-oxydase; NRG, neuregulin; ROS, reactive oxygen species; Smad, Small-mothers against decapentaplegic; TGF, transforming growth factor; TGFBR, TGF $\beta$ receptor. 


\section{The transforming growth factor $\beta$ (TGF- $\beta$ ) superfamily}

Members of the transforming growth factor $\beta$ (TGF- $\beta$ ) superfamily are highly conserved across animals. They are found in both vertebrates and invertebrates, are ubiquitously expressed in diverse tissues and function during the earliest stages of development and throughout life. The TGF- $\beta$ superfamily of secreted factors comprises more than 30 members including activins, nodals, bone morphogenetic proteins (BMPs), and growth and differentiation factors (GDFs). TGF- $\beta$ family members are involved in a wide range of diverse functions and play key roles in embryogenesis, development and tissue homeostasis. TGF- $\beta$ superfamily signaling is associated with a wide range of human pathologies including autoimmune and cardiovascular diseases, and cancer (Pardali \& Ten Dijke, 2012). Recently, myostatin, a cytokine from the TGF- $\beta$ family, has been identified as a direct mediator of skeletal muscle atrophy in mice with heart failure (Biesemann, et al., 2014).

Members of the TGF- $\beta$ superfamily exert their effects by binding to specific serine/threonine kinase type I and type II receptor complexes. These receptors, known as TGF- $\beta$ type I and type II receptors, or T $\beta R-I$ and TR-II, are structurally similar and include small disulfide-rich ectodomains that adopt a three-finger toxin fold ( $\sim 120$ residues), single-spanning transmembrane domains ( $\sim 30$ residues), and cytoplasmic serine-threonine kinase domains ( 400 residues) (Hinck, 2012). In mammals, seven type I receptors, also termed activin receptor-like kinase (ALK) 1 to 7 and five type II receptors have been identified. On the formation of heteromeric complex between type I and type II receptors, the type I receptor is transphosphorylated by the type II receptor. A conformational change is associated with the activation of the type I receptor, which can subsequently propagate the signal inside the cell by the phosphorylation of specific effectors. In endothelial cells, however, TGF- $\beta$ has been shown to bind and signal via both ALK-1 and ALK-5 (Bertolino, Deckers, Lebrin, \& ten Dijke, 2005; Brown \& Schneyer, 2010).

ALK-1 interacts with four ligands: TGF- $\beta 1$ and TGF- $\beta 3$, in a complex with the receptor type II); and with Bone Morphogenetic Potein 9 (BMP-9) and BMP-10, in a complex with the activin receptor type IIA (ActRIIA) or the BMP receptor type II (BMPRII). ALK-1 activation induces phosphorylation of Smad1/5/8, which in turn dimerizes with a common partner, Smad4. This complex then translocates to the nucleus and directly regulates transcription of specific genes. Smad7 antagonizes TGF- $\beta$ signaling through multiple mechanisms both in the cytoplasm and in the nucleus. In humans, ALK-1 mRNA was first described as being expressed in the placenta, adipose tissue and skeletal muscle. Its expression was shown to be more prominent in endothelial cells and to a lesser extent in vascular smooth muscle cells, and it appears to be restricted to the arteries (Gonzalez-Nunez, Munoz-Felix, \& Lopez-Novoa, 2013).

BMPs are typically divided into at least four subgroups: BMP2/ 4, BMP5/ 6/ 7/ 8a/ 8b, BMP9/ 10, and BMP12/ 13/ 14. BMPs are crucial growth factors that regulate numerous processes of skeletal formation, hematopoesis, neurogenesis, and differentiation of cells during embryonic development. The BMP pathway also plays important roles in the adult vascular endothelium, where it promotes angiogenesis and mediates shear and oxidative stress. Deregulated BMP signaling has been linked to vascular diseases including pulmonary hypertension and atherosclerosis (Dyer, Pi, \& Patterson, 2014).

Myostatin is mainly expressed in skeletal muscle, although basal expression is also detectable in heart and adipose tissue. Gene expression data suggest that the levels of myostatin signaling 
pathway genes are altered in adipose tissue in response to obesity. The metabolic changes found in myostatin-deficient animals raise the possibility that myostatin inhibition could be used to treat metabolic diseases such as obesity and diabetes. The inhibition of myostatin protein function in adult mice also increases muscle mass. These results suggest that anti-myostatin therapy could be used as a treatment for muscle wasting diseases (Patel \& Amthor, 2005). The expression of myostatin in cardiomyocytes implies its involvement in physiological as well as pathophysiological cardio-vascular processes. Moreover, myostatin may promote fibrosis and left ventricular hypertrophy (Breitbart, Auger-Messier, Molkentin, \& Heineke, 2011). In addition to myostatin, a Drosophila myostatin (myoglianin) extends lifespan and delays systemic aging (Demontis, Patel, Swindell, \& Perrimon, 2014).

The access of ligands to the signaling receptors is regulated by numerous secreted agonists and antagonists and by membrane-associated coreceptors. Among the different ligands, activins have been implicated in the development and maintenance of various organs, in which stem cells play important roles. The first-identified and best-studied family member is activin A; activins are highly conserved across species (Hedger \& de Kretser, 2013).

As mentioned above, TGF- $\beta$ family ligands bind and activate specific heteromeric type I and type II Ser/Thr kinase receptor complexes, which propagate the signal by phosphorylating receptor regulated (R)-Smads (Figure 1). Two distinct R-Smad pathways exist: the TG-F $\beta$-Smad pathway (RSmad2/3) and the BMP-Smad pathway (R-Smad1/5/8) (Massague, 1998). Members of the Smad family of transcription factors are important intracellular messengers. TGF- $\beta$ signaling is tightly controlled by post-translational modifications, which regulate the activity, stability, and subcellular localization of the signaling components. The three-dimensional structure of TGF- $\beta$ monomers is like a hand. Two monomers are organized in an antiparallel manner, and the dimer is stabilized by a disulfide bond. The ligand receptor complex consists of one dimeric ligand and two units each of T $\beta R$ I and T $\beta R$-II. In the case of BMPs, the ligand binds independently to type I and type II receptors, and most of the binding affinity comes from interaction with the type I receptor (Bragdon, et al., 2011). As mentioned above, activins bind to one of two specific type 2 activin receptors on the cell surface. Several molecules act to interfere with the ability of activins to activate their receptors. The inhibins, $A$ and $B$, are able to bind to the type 2 activin receptor subunit through the mediation of a coreceptor called TGF $\beta$ receptor 3 (TGFBR3), or betaglycan, thus preventing the dimerisation of the type 2 and type 1 receptor, which is necessary to initiate intracellular signaling. Inhibition of activin action can reduce inflammation, tissue damage, fibrosis and morbidity/mortality in various experimental models. Endogenous activin-binding proteins, such as follistatin, offer considerable promise as therapies. Follistatin is a potential mediator of inflammation and insulin resistance in obesity. The synthesis and release of activins are stimulated by inflammatory cytokines, Toll-like receptor ligands and oxidative stress. Follistatin is an important biological regulator of activin activity in vivo, and this is believed to be its principal biological activity. However, follistatin also binds with lower affinity to several other members of the TGF- $\beta$ superfamily (Fan, et al., 2013).

\section{GDF11 biology}

GDF11, like other members of the TGF- $\beta$ superfamily, are produced from precursor proteins by proteolytic processing. After cleavage of a single peptide bond by a furin-type protease, the $\mathrm{N}$ - 
terminal propeptide and the disulfide-bonded homodimer containing the mature growth factor domains remain associated, forming an inactive complex known as the latent complex (Figure 2). The active mature growth factors may be liberated from the latent complexes through degradation of the propeptide by a variety of proteases (Kondas, Szlama, Nagy, Trexler, \& Patthy, 2011). Different functions of GDF11 in controlling progenitor proliferation and/or differentiation have been demonstrated (Kim, et al., 2005). In the brain, it has been demonstrated that GDF11 signals produced by newly differentiated neurons act as a feedback signal on the adjacent progenitors to promote cell cycle exit, and facilitate temporal progression of neurogenesis (Shi \& Liu, 2011).

As we mentioned previously, members of the TGF-beta superfamily signal through a combination of type I and type II receptors, both of which are transmembrane serine/threonine kinases. GDF11 interacts with the type I receptors: ALK-7 (ACVR1C), and ALK-4 (ACVR1B) and activin receptor type II (type II receptors: ActRIIA, ActRIIB or ACVR2). The activin type II receptor has an extracellular ligand binding domain, a single transmembrane domain and the intracellular serine/threonine kinase domain. Signaling through activin receptors could be a therapeutic target in multiple diseases (Tsuchida, Nakatani, Uezumi, Murakami, \& Cui, 2008). Several GDF11 binding proteins have been identified. These include GDF-associated serum protein-1 (GASP-1) and GASP-2, which are capable of inhibiting the activities of these ligands. Both GASP-1 and GASP-2 are important modulators of GDF11 activity in vivo (Lee \& Lee, 2013). GASP-1 is a large acidic protein of 1,394 amino acids that is highly expressed in a several tissues. The 497-amino-acid COOH terminal of GASP-1 (cGASP-1) can disrupt the interaction of GASP-1 with 7-transmembrane-spanning (7TM) G protein-coupled receptors (GPCR). It is possible that though GASP-1 and GASP-2 have similar functions, GASP-1 is a potential new serum and tumor biomarker for breast cancer. It may be a novel target for the development of breast cancer therapeutics (Tuszynski, et al., 2011). Recent studies have investigated new compounds: 1) ACE-536, a receptor fusion protein consisting of a modified human ActRIIB extracellular domain linked to the human IgG1 Fc domain, 2) RAP-536, a mouse version of ACE-536 with an identical ligand-binding domain. ACE-536 and RAP-536 bound GDF11 and potently inhibited GDF11-mediated Smad2/3 signaling (Suragani, et al., 2014). Sotatercept (formerly known as ACE011) (fusion protein of Human soluble Activin receptor type-IIA and Fc-portion of human IgG1) is another ligand trap that has been shown to inhibit inhibitory Smad2/3 signaling (Raje \& Vallet, 2010). Inhibiting GDF11 signaling with compounds such as ACE-536 or sotatercept promotes apoptosis in cells that express this growth factor (Figure 1).

As mentioned above, signaling by TGF- $\beta$ family ligands is essential for the regulation of multiple processes during vertebrate development, tissue homeostasis, and tissue repair. In the mouse, the Smad2-activating ligand GDF11 has been shown to be essential for anterior-posterior patterning. Studies in mice have shown that GDF11 is important for generating correct anterior be essential for anterior-he treatment of anemia and bone loss (Ho, Yeo, \& Whitman, 2010). Recently, it has been demonstrated that treatment with SM16, an orally active small molecular inhibitor of ALK-5, in mice subjected to left ventricular pressure overload for 4 weeks, attenuates the development of cardiac fibrosis and collagen cross-linking. SM16 abolished phosphorylation of Smad2 induced by aortic binding in vivo and by TGF- $\beta$ in cardiac fibroblasts in vitro (Engebretsen, et al., 2014). ALK-1 is mainly expressed in endothelium, whereas ALK-5 is mainly in smooth muscle cells. The role of ALK-1 in inflammatory cells seems to be significant and the expression of ALK-1 in endothelial cells has been shown to increase proliferation and migration (Gonzalez-Nunez, et al., 2013). Studies demonstrated 
that TGF- $\beta$ plays an important role in the pathogenesis of the infarcted heart due to inflammatory and reparative response modulation (Bujak, et al., 2007).

Mechanisms by which TGF- $\beta$ may maintain cellular homeostasis is through the induction of cytoprotective proteins such as heme-oxygenases-1 (HO-1) and/or interaction with NADPH-oxydases (Nox) or nitric oxide synthases (NOS). The HOs are rate-limiting enzymes that catalyze the conversion of heme into carbon monoxide, free iron and biliverdin, which is subsequently converted to bilirubin by biliverdin reductase. The products of the $\mathrm{HO}$ reaction modulate important adaptive responses to oxidative stress, inflammation, and cardiovascular functions (Rochette, Cottin, Zeller, \& Vergely, 2013). HO-1 may be an adaptive response that provides a balance between the effects of growth factors. Conversely, it is possible that some of the effects associated with growth factors may be attributable to HO-1 induction (Hill-Kapturczak, Jarmi, \& Agarwal, 2007). Concerning the relationship between Nox activities and TGF- $\beta$, it has been demonstrated that Nox4-mediated TGF $\beta$-1-induced conversion of fibroblasts to myofibroblasts by regulating Smad2/3 activation (Barnes \& Gorin, 2011). Nox are enzymes that generate superoxide by transferring electrons from NADPH to molecular oxygen. The Nox family is defined by seven distinct membrane-integrated catalytic subunits that form the basis of the enzyme. Nox4, originally described in the kidney, has a more ubiquitous expression profile, including smooth muscle cells, fibroblasts, hematopoietic stem cells, osteoclasts, neurons, and endothelial cells. Within the cell, Nox4 has been detected near focal adhesions, in the endoplasmic reticulum, in the nucleus, and in the mitochondria, depending on the cell type. Nox subunit expression and activity are increased in various models of cardiac hypertrophy and heart failure. Complex cellular and inflammatory interactions are involved in the advance of vascular diseases. Endothelial cells, upon exposure to cytokines, undergo profound alterations of function that involve gene expression and de novo protein synthesis. Endothelial NOS (eNOS) is a key enzyme responsible for the production of NO by the endothelium. Its expression and activity are very important with respect to endothelial protection, and its decreased expression is related to the development of endothelial dysfunction. Several TGF- $\beta$-mediated mechanisms may cause modifications of endothelial functions. TGF- $\beta$ receptor expression was related to the expression of eNOS in the endothelium. TGF- $\beta$ inhibits the production of NO in macrophages and the endothelium (Rochette, Lorin, et al., 2013).

\section{GDF11 and erythropoiesis}

A complex interplay of various cytokines is involved in maintaining normal hematopoiesis. The process of differentiation of hematopoietic stem cells into mature blood cells is tightly regulated by the actions of both stimulatory and inhibitory cytokines. The stimulatory role of erythropoietin (EPO) in erythropoiesis is well established. Anemia can result from direct defects, as seen in congenital anemia or by indirect causes, as is the case in anemia associated with persistent inflammatory disease, for example. It leads to tissue hypoxia, which, in turn, increases EPO production by the kidney, which stimulates bone marrow erythropoiesis. The vicious cycle is associated with changes in iron utilization caused by the decreased expression of hepcidin, the hormone that regulates iron uptake (Rochette, et al., 2014). Recent studies suggest a new pathway for the treatment of anemia. This pathway involves targeting a newly discovered regulator of erythropoiesis via GDF11. These studies showed that GDF11-ActRIIB-Smad2/3-dependent signaling is a key regulatory mechanism in 
proliferating erythroid precursors that controls their late-stage maturation under both steady-state and stress conditions. The findings raise the possibility that TGF- $\beta$ superfamily signaling pathways may become new EPO-independent therapeutic targets. The results indicate that GDF11 negatively regulates late-stage erythroid differentiation. This is supported by the finding that chronic administration of GDF11 to wild-type mice produced mild anemia. However, ligand traps such as ACE-536, or its mouse version RAP-536, which inhibit GDF11-mediated Smad2/3 signaling, produced rapid increases in erythrocyte numbers and reduced or prevented anemia in murine models. Cotreatment with ACE-536 and EPO produced a synergistic erythropoietic response (Suragani, et al., 2014).

Sotatercept, the ligand trap that inhibits inhibitory $\mathrm{Smad} 2 / 3$ signaling, was originally developed to treat bone-loss disorders, but clinical studies revealed unexpected effects of the drug, including increased hematocrit and hemoglobin levels (Ruckle, et al., 2009). It has recently been demonstrated that treatment with a mouse version of sotatercept (RAP-011) 1) reverses ineffective erythropoiesis in thalassemic mice and that the erythropoietic effects of this ligand trap are due to inactivation of the ActRIIA ligand GDF11, 2) blocks terminal erythroid maturation through an autocrine amplification loop involving oxidative stress via reactive oxygen species (ROS). On the other hand, higher GDF11 expression has been demonstrated in spleens from thalassemic mice than in those from wild-type control mice. Treatment of thalassemic mice with RAP-011 decreased the expression of GDF11 (Dussiot, et al., 2014). In addition to anemia, ineffective erythropoiesis in subjects with $\beta$-thalassemia results in systemic iron overload, increased gastrointestinal iron absorption and decreased circulating levels of hepcidin levels. RAP-011 decreased the iron overload induced by beta-thalassemia. In recent experimental studies, it was demonstrated that hepatic hepcidin mRNA levels were higher in RAP011-treated mice than in PBS-treated controls. Recent data support the hypothesis that RAP-011 is able to prevent depletion of mouse splenic iron stores. RAP-011 may therefore be an appropriate therapy for trials in human anemia characterized by increased expression of hepcidin and ironrestricted erythropoiesis (Langdon, et al., 2015).

It is important to note the importance of iron metabolism and oxidative stress in the erythroid processes associated with anemia. Hepcidin synthesis is increased by high iron stores and inflammation, while iron deficiency, hypoxia and active erythropoiesis suppress hepcidin levels. As red blood cell (RBC) formation is the main iron consumer of the body, it was supposed that hepcidin was directly regulated by the intensity of erythropoietic activity, even though the mediators are not known. Compounds such as GDF15 or GDF11 were also reported to play a role (Paulson, 2014; Tanno, et al., 2007) (Figure 3).

Oxidative stress promotes terminal erythroid maturation arrest in healthy erythroblasts. Forkhead box 03 (Foxo3) translocates to the nucleus and regulates the transcription of anti-oxidant enzymes in mammalian cells, Foxo3 being a critical physiological regulator of oxidative stress. Foxo3-deficient erythrocytes exhibited decreased expression of ROS- scavenging enzymes and had an ROS-mediated shortened lifespan (Marinkovic, et al., 2007). It is important to remember the relationship between oxidative stress and hematological processes. In this field, the mechanisms of cellular protection responses to EPO have been investigated. EPO may act indirectly by inducing iron depletion and thereby inhibiting iron-dependent oxidative injury. EPO has been shown to exert cytoprotective effects on erythroid progenitor cells as well as various non-erythroid cells. EPO is required and necessary for the growth, survival and differentiation of red blood progenitor cells. EPO exerts its 
physiological role by binding to its specific cell surface receptor (EpoR). EPO has been used widely for the treatment of cancer patients with chemotherapy-induced anemia. In these clinical trials, treatment with EPO increased RBC levels and hemoglobin concentrations. It also significantly reduced ROS production in polymorphonuclear leukocytes, which may contribute to its antiinflammatory effect (Wang, Di, \& Noguchi, 2014).

Since oxidative stress plays a key role in the pathogenesis of $\beta$-thalassemia, the use of various molecules with antioxidant properties as possible therapeutic strategy in $\beta$-thalassemia has been explored. A pilot trial with large doses of oral vitamin E, prompted by the abnormally low levels of this vitamin in the plasma of $\beta$-thalassemia intermedia patients, showed a decrease in the levels of malonylaldehyde, an index of lipid peroxidation (Tesoriere, et al., 2001). Low-dose resveratrol, a polyphenolic-stilbene with antioxidant and anti-inflammatory properties, induces early maturation of normal erythroid precursors by activation of the Foxo3 transcriptional factor, inhibition of Akt and upregulation of antioxidant response genes such as catalase. The effects of resveratrol on cell maturation are highly dependent on resveratrol concentrations and on cell types (Jeong, et al., 2011). Recently, the beneficial effects of resveratrol supplementation on pathological erythropoiesis have been reported in a mouse model of Fanconi's anemia (FA), which is characterized by the hypersensitivity of FA cells to ROS. Finally, resveratrol might be considered a new possible complementary agent in the treatment of anemia of $\beta$-thalassemia. Its properties imply the existence of a novel mechanism that promotes terminal erythroid differentiation associated with the activation of Foxo3 and the upregulation of anti-oxidant systems (Franco, et al., 2014).

Clinical studies in healthy subjects revealed that sotatercept rapidly increases red blood cell counts, hemoglobin levels and hematocrit (Ruckle, et al., 2009). ACE-536 and sotatercept have completed phase 1 clinical studies in healthy volunteers, and phase 2 studies are ongoing with these agents for anemia in patients with thalassemia (Sherman, et al., 2013).

\section{GDF11 and cardiac regeneration}

Aging is associated with an extensive loss of function at all levels of biological organization. Studies using model organisms have generated significant insights into the genetic factors and environmental conditions that influence the age-related decline (Demontis, Piccirillo, Goldberg, \& Perrimon, 2013; Wang, Karpac, \& Jasper, 2014). Progressive aging induces several structural and functional alterations in the cardiovascular system. The most important of these include a reduced number of myocardial cells and increased interstitial collagen fibers, which result in the development of heart failure. The signals and mechanisms that cause age-related tissue failure are unclear. In the cardiovascular field, the treatments can be cardioprotective [protecting heart muscle tissue after an acute myocardial infarction (MI) and limiting the progress of coronary heart failure (CHF)], or cardiorestorative (regenerating tissue in patients with these diseases). Post-infarction remodeling and the progression to heart failure remain a challenge in the treatment of cardiovascular diseases. Tissue regeneration is characterized by complex cascades of growth factors with critical roles in cell proliferation and differentiation. The combination of several growth factors is required to imitate the native environment and stimulate the formation of new functional tissue (Segers \& Lee, 2010). It is important for the therapeutic formulations to reach the region of the infarcted myocardium where they are required. Cardiac regenerative therapy, whereby pro-regenerative cells, drugs or growth 
factors are administered to damaged and ischemic myocardium, has demonstrated significant potential, especially in a preclinical context. However, the transfer to clinical use remains disappointing (Hastings, et al., 2014). Cell-based strategies for cardiac repair involve delivering cells with potential for repair or regeneration to ischemic or damaged areas of the heart (Korf-Klingebiel, et al., 2008). Multiple trials have been initiated to investigate the transplantation of stem-cell populations for cardiac regeneration. Extensive preclinical and clinical trials have investigated a number of cell types for cardiac regeneration including skeletal myoblasts, mesenchymal stem cells, embryonic stem cells and cardiac stem cells. Although most cell types had produced promising results in vitro and in preclinical studies, they were disappointing in terms of clinical benefits (Haider, Lei, \& Ashraf, 2008; Sanganalmath \& Bolli, 2013).

Small molecule drugs, peptides and proteins represent a promising therapeutic deliverable for the treatment of cardiomyopathy and heart failure (Jung \& Williams, 2012). Early clinical studies have also been performed with recombinant human neuregulin-I (NRG-I), a member of the epidermal growth factor family that promotes increased cell cycle activity and proliferation of cardiomyocytes through ErbB4 receptor binding (Jabbour, et al., 2011). Prostaglandin E2 and Prostaglandin 12 play a regenerative role in the ischemic myocardium and may have therapeutic potential in a postmyocardial infarction setting (Hsueh, Wu, $\mathrm{Yu}, \mathrm{Wu}, \&$ Hsieh, 2014). In addition to its main role in hematopoiesis, EPO presents antiapoptotic and pro-angiogenic properties that have shown efficacy against myocardial infarction in different animal models. Clinical studies, however, failed to show significant therapeutic efficacy (Sanchis-Gomar, et al., 2014).

In a very interesting new study, it was demonstrated that GDF11 was a circulating negative regulator of cardiac hypertrophy, suggesting that raising GDF11 levels could potentially treat or prevent agerelated cardiac hypertrophy (Loffredo, et al., 2013) (Figure 1). The authors used parabiosis to determine whether a specific type of cardiac hypertrophy related to aging was due to age-related differences in levels of a factor or factors in the blood. Parabiosis [from the Greek "para" (next to) and "bios" (life)] refers to the condition in which two entire living animals are joined surgically and develop a single, shared circulatory system. Over time, the procedure has improved and, in the early 70s, scientists started to graft animals of different ages to each other. This heterochronic parabiosis set the basis for the investigation of the effects induced through exposure of an aged organism to a youthful systemic environment (Conboy, Conboy, \& Rando, 2013; Eggel \& Wyss-Coray, 2014). Leffredo et al (Loffredo, et al., 2013) generated parabiotic pairs of young with old (heterochronic) mice and compared their heart sizes with those of parabiotic pairs of mice of the same age (isochronic) and those of age-matched controls that did not undergo parabiosis. After only 4 weeks, cardiac hypertrophy was reversed in the old heterochronically paired mice. Importantly, these effects were gender-independent and did not arise from the parabiosis technique itself, or changes in blood pressure. The authors identified that the myocyte cross-sectional area was decreased in aged mice paired with younger adult mice. These results are consistent with the loss of youthful factors that restrict myocyte size in the aged systemic environment rather than the accumulation of hypertrophic factors during ageing. They also investigated the molecular nature of cardiac hypertrophy using various molecular markers of cardiac hypertrophy The authors compared levels of molecules found in the circulation of old versus young mice using a proteomic approach and they showed that levels of GDF11 were constantly lower in aged than in younger adult plasma. To demonstrate that GDF11 was a negative regulator of cardiac myocyte hypertrophy, these authors injected recombinant GDF11 daily for 30 days in aged mice using a blinded, randomized study design. Heart weight and 
cardiomyocyte area were reduced without affecting cardiac function. The function of GDF11 in cardiac muscle is analogous to that of another TGF- $\beta$ superfamily member, myostatin, also known as GDF8. The amino acid sequence of GDF11 is $90 \%$ identical to that of myostatin (Dschietzig, 2014).

GDF11 has been measured in different tissues. It is expressed in the pancreas, intestine, kidney, skeletal muscle, heart and developing nervous system, olfactory system and retina (McPherron, 2010). Its expression was most abundant in young adult organs and decreased during ageing. GDF11 affects multiple aspects of spinal cord development (Shi \& Liu, 2011). GDF11 secreted by newly born neurons has multiple effects on neighboring progenitor cells during neurogenesis. These effects of GDF11 in the developing spinal cord are similar to those observed in the olfactory epithelium and developing retina. GDF11 has diverse positive effects in aging mice, including enhanced neurogenesis. In vitro experiments confirmed that GDF11 acts on brain capillary endothelial cells. Treatment of endothelial cells with rGDF11 activates the TGF- $\beta$ signaling pathway in these cells, thus stimulating the Smad phosphorylation cascade. The treatment of primary brain capillary endothelial cells with rGDF11 increased their proliferation as compared with that of controls but not in the presence of a TGF- $\beta$ inhibitor, thus confirming that GDF11 has a direct biological effect on these cells through the Smad pathway (Katsimpardi, et al., 2014).

Evidence indicates that skeletal muscle influences systemic aging, but little is known about the signaling pathways. It has been suggested that muscle was a key tissue with the ability to influence systemic aging and lifespan. Activation of the transcription factor Foxo in skeletal muscles preserves muscle function and extends lifespan (Bai, Kang, Hernandez, \& Tatar, 2013). Skeletal muscle is a highly specialized tissue composed predominantly of contractile fibers whose regeneration depends on the activity of a specialized subset of muscle fiber-associated mononuclear stem cells called satellite cells. Aged muscle exhibits decreased satellite cell number and impaired satellite cell function. Several growth factors and cytokines, such as TGF- $\beta$ and myostatin, are regulators of muscle growth and repair (Brooks \& Myburgh, 2014; Burks \& Cohn, 2011). rGDF11 supplementation in aged mice enhanced the regenerative capacity of satellite cells. Recent studies reported that GDF11 is a humoral regulator of youthful regenerative potential and demonstrated that the restoration of aged satellite cell function by GDF11 was associated with the reversal of accumulated DNA damage (Sinha, et al., 2014).

The regulation of members of the TGF- $\beta$ family is considered complex, because many proteins able to bind the ligands and inhibit their activities have been identified (Souza, et al., 2008). One of these binding proteins is GDF-associated serum protein-1 GASP-1, also known as WFIKKNRP or WFIKKN2, which was isolated from mouse and human serum as a myostatin-associated protein. GASP-1 contains many conserved domains associated with protease-inhibitory proteins, and GASP-1 is closely related to GASP-2 (also known as WFIKKN or WFIKKN1). Overexpression of GASP-1 in mice has been shown to cause increased muscle growth. GASP-1 and GASP-2 are important modulators of GDF-11 and myostatin activity in vivo (Kondas, et al., 2011; Kondas, Szlama, Trexler, \& Patthy, 2008; Lee \& Lee, 2013). In summary, GDF11 appears to be a modulator of age-related cardiac hypertrophy. It is suggested that GDF11 supplementation in older patients may represent a new approach for the treatment of some cardiac and skeletal diseases. 


\section{Conclusion and perspectives: GDF-11 and CCL11 in young blood: growth factors that "rejuvenate" the heart and brain?}

As we reported previously, in comparing the proteomic profiles of blood samples from young and old mice, GDF11 was found to be greatly far lower in the bloodstream of old mice than in that of young mice. The reduced circulating levels of GDF11 observed in old mice may contribute to cardiac aging. GDF11 administration is effective in experimental cardiac hypertrophy. The identification of GDF11 as a "rejuvenating factor" therefore opens up perspectives for the treatment of age-related cardiac dysfunction (Loffredo, et al., 2013). These studies provide further evidence for the potentially rejuvenating effects of young blood. A related question is whether or not continuous treatment is required to rejuvenate tissues of aged mice.

Recent studies have identified a second factor in mouse blood that mediates rejuvenating actions; it is a chemokine (C-C motif) chemokine 11: CCL11 (Villeda, et al., 2014). CCL11 is also known as eosinophil chemotactic protein. In contrast to GDF11, levels of CCL11 increase with age, and this increase appears to contribute to the decline in neurogenis and function in neural stem cells in brain areas such as the hippocampus. CCL11 has high selectivity for the chemokine receptors CCR3, and these receptors are expressed on endothelial cells of the blood-brain barrier (Erickson, Morofuji, Owen, \& Banks, 2014). There is also increasing evidence that chemokine receptor expression in the brain and the medulla increases during pathological conditions, especially inflammation. Increased circulating levels of CCL11 have been implicated in diseases associated with abnormal central nervous system function, including Alzheimer disease (Leung, et al., 2013). The administration of young blood plasma improves hippocampal-dependent learning and memory in aged mice. These recent data indicate that the administration of soluble heat-labile factors from young blood "rejuvenates" cognitive function in aged animals. The discovery of systemic factors that appear to modulate aging, such as GDF11 and CCL11, has potential major therapeutic implications. GDF11 and CCL11 are perhaps only the first two in a series of circulating molecules that will be found to influence aging of different tissues. Are CCL11 and GDF11 endogenous factors for an "elixir of youth"? 


\section{Acknowledgments}

The authors wish to thank Martine Goiset for secretarial assistance and Philip Bastable for English assistance.

\section{Funding}

This work was supported by grants from the French Ministry of Research, from the Institut National de la Santé et de la Recherche Médicale (INSERM) and from the Regional Council of Burgundy. 


\section{Legend to Figures}

Figure 1: Schematic representation of rejuvenation process of heart and erythropoiesis with GDF11 and activing production signaling.

Members of TGF- $\beta$ superfamily exert their effects by binding to specific serine/threonine kinase type I and type II receptors complexes: activing receptor -like-kinase (ALK). (type I receptors: ALK-7; ALK-4 and activing receptor type II:ACVR). ALK-1 activation induces phosphorylation of Smads. Smad 7 antagonizes TGF- $\beta$ signaling. The development of red blood cells in bone marrow is regulated by hormone erythropoietin (EPO) that stimulates the differentiation of pluripotent stem cells into erythroid progenitors. GDF11 negatively regulates late-stage erythroid differentiation. Rejuvenation: Linking an old mouse to the circulation of a young mouse or injecting the animal with GDF-11 reversed signs of aging cardiac muscle.

\section{Figure 2: Schematic overview of TGF-B secretion and activation.}

TGF-B associated with Latent Transforming Growth Factor B - Binding Protein (LTBP) inside the cell form the Large Latent Complex (LLC). TGF- $\beta$ is secreted in a latent dimeric complex containing the Cterminal mature TGF- $\beta$ and its $\mathrm{N}$-terminal pro-domain, LAP (TGF- $\beta$ latency associated protein). The two polypeptide chains of pro-TGF-B associate to form a disulphide bonded dimer involving Cys-33 of LAP. Cysteine residues are required for the dimerization of LAP and thus for TGF- $\beta$ inactivation. Proteolytic activation (plasmin, MMPs, thrombospondin-1, integrins) and, ROS is associated with TGF- $\beta$ activation mechanism. Members of the TGF- $\beta$ family are only active as dimers. The TGF- $\beta$ monomers contain four intrachain disulphide bonds. The dimers are bridged by one intermolecular disulphide bond. The LAP peptide prevents the interaction between TGF- $\beta$ and its receptors.

\section{Figure 3: Regulation of systemic iron metabolism and regulatory mechanisms}

Hepatocytes store iron, and play a crucial role in iron metabolism by producing transferrin, the iron carrier protein, and hepcidin, a hormone involved in regulating iron metabolism. The synthesis and secretion of hepcidin by hepatocytes are influenced by iron levels in relationship with infection and inflammation. Hepcidin and a new hormone: erythroferrone play a central role in the maintenance of iron homeostasis and regulation of plama iron concentration by controlling ferroportin levels on ironexporting cells. Hepcidin inhibits the release of iron from enterocytes of the duodenum and from macrophages by binding to the iron exporter protein, ferroportin (FPN). Splenic macrophages are specialized for iron recycling with increased expression of proteins for the uptake of hemoglobin (transferrin receptors: TFR), breakdown of heme and the export of iron via ferroportins (FPN). Heme is degraded through the action of heme oxygenase-1 (HO-1) to release iron. Erythroferrone is produced by erythroid precursors in the bone marrow and the spleen and is able to suppress hepcidin expression. Two members of TGF- $\beta$ family play roles in these regulatory mechanisms: GDF11 and GDF15. GDF11 acts as a key regulatory mechanism in proliferating erythroid precursors, controlling their late-stage maturation. GDF15 can promote the growth and differentiation of 
erythroid progenitors, it contributes to the suppression of hepcidin and subsequent tissue iron overloading. 


\section{References}

Bai, H., Kang, P., Hernandez, A. M., \& Tatar, M. (2013). Activin signaling targeted by insulin/dFOXO regulates aging and muscle proteostasis in Drosophila. PLoS Genet, 9, e1003941.

Barnes, J. L., \& Gorin, Y. (2011). Myofibroblast differentiation during fibrosis: role of NAD (P)H oxidases. Kidney Int, 79, 944-956.

Bertolino, P., Deckers, M., Lebrin, F., \& ten Dijke, P. (2005). Transforming growth factor-beta signal transduction in angiogenesis and vascular disorders. Chest, 128, 585S-590S.

Biesemann, N., Mendler, L., Wietelmann, A., Hermann, S., Schafers, M., Kruger, M., Boettger, T., Borchardt, T., \& Braun, T. (2014). Myostatin regulates energy homeostasis in the heart and prevents heart failure. Circ Res, 115, 296-310.

Bragdon, B., Moseychuk, O., Saldanha, S., King, D., Julian, J., \& Nohe, A. (2011). Bone morphogenetic proteins: a critical review. Cell Signal, 23, 609-620.

Breitbart, A., Auger-Messier, M., Molkentin, J. D., \& Heineke, J. (2011). Myostatin from the heart: local and systemic actions in cardiac failure and muscle wasting. Am J Physiol Heart Circ Physiol, 300, H1973-1982.

Brooks, N. E., \& Myburgh, K. H. (2014). Skeletal muscle wasting with disuse atrophy is multidimensional: the response and interaction of myonuclei, satellite cells and signaling pathways. Front Physiol, 5, 99.

Brown, M. L., \& Schneyer, A. L. (2010). Emerging roles for the TGFbeta family in pancreatic beta-cell homeostasis. Trends Endocrinol Metab, 21, 441-448.

Bujak, M., Ren, G., Kweon, H. J., Dobaczewski, M., Reddy, A., Taffet, G., Wang, X. F., \& Frangogiannis, N. G. (2007). Essential role of Smad3 in infarct healing and in the pathogenesis of cardiac remodeling. Circulation, 116, 2127-2138.

Burks, T. N., \& Cohn, R. D. (2011). Role of TGF-beta signaling in inherited and acquired myopathies. Skelet Muscle, 1, 19.

Conboy, M. J., Conboy, I. M., \& Rando, T. A. (2013). Heterochronic parabiosis: historical perspective and methodological considerations for studies of aging and longevity. Aging Cell, 12, 525530.

Demontis, F., Patel, V. K., Swindell, W. R., \& Perrimon, N. (2014). Intertissue control of the nucleolus via a myokine-dependent longevity pathway. Cell Rep, 7, 1481-1494.

Demontis, F., Piccirillo, R., Goldberg, A. L., \& Perrimon, N. (2013). The influence of skeletal muscle on systemic aging and lifespan. Aging Cell, 12, 943-949.

Dschietzig, T. B. (2014). Myostatin - From the Mighty Mouse to cardiovascular disease and cachexia. Clin Chim Acta, 433, 216-224.

Dussiot, M., Maciel, T. T., Fricot, A., Chartier, C., Negre, O., Veiga, J., Grapton, D., Paubelle, E., Payen, E., Beuzard, Y., Leboulch, P., Ribeil, J. A., Arlet, J. B., Cote, F., Courtois, G., Ginzburg, Y. Z., Daniel, T. O., Chopra, R., Sung, V., Hermine, O., \& Moura, I. C. (2014). An activin receptor IIA ligand trap corrects ineffective erythropoiesis in beta-thalassemia. Nat Med, 20, 398-407.

Dyer, L. A., Pi, X., \& Patterson, C. (2014). The role of BMPs in endothelial cell function and dysfunction. Trends Endocrinol Metab, 25, 472-480.

Eggel, A., \& Wyss-Coray, T. (2014). A revival of parabiosis in biomedical research. Swiss Med Wkly, 144, w13914.

Engebretsen, K. V., Skardal, K., Bjornstad, S., Marstein, H. S., Skrbic, B., Sjaastad, I., Christensen, G., Bjornstad, J. L., \& Tonnessen, T. (2014). Attenuated development of cardiac fibrosis in left ventricular pressure overload by SM16, an orally active inhibitor of ALK5. J Mol Cell Cardiol, 76, 148-157.

Erickson, M. A., Morofuji, Y., Owen, J. B., \& Banks, W. A. (2014). Rapid transport of CCL11 across the blood-brain barrier: regional variation and importance of blood cells. J Pharmacol Exp Ther, 349, 497-507. 
Fan, N., Sun, H., Wang, Y., Zhang, L., Xia, Z., Peng, L., Hou, Y., Shen, W., Liu, R., Yin, J., \& Peng, Y. (2013). Follistatin-like 1: a potential mediator of inflammation in obesity. Mediators Inflamm, 2013, 752519.

Franco, S. S., De Falco, L., Ghaffari, S., Brugnara, C., Sinclair, D. A., Matte, A., Iolascon, A., Mohandas, N., Bertoldi, M., An, X., Siciliano, A., Rimmele, P., Cappellini, M. D., Michan, S., Zoratti, E., Anne, J., \& De Franceschi, L. (2014). Resveratrol accelerates erythroid maturation by activation of $\mathrm{FoxO}_{3}$ and ameliorates anemia in beta-thalassemic mice. Haematologica, 99, 267-275.

Gonzalez-Nunez, M., Munoz-Felix, J. M., \& Lopez-Novoa, J. M. (2013). The ALK-1/Smad1 pathway in cardiovascular physiopathology. A new target for therapy? Biochim Biophys Acta, 1832, 1492-1510.

Haider, H., Lei, Y., \& Ashraf, M. (2008). MyoCell, a cell-based, autologous skeletal myoblast therapy for the treatment of cardiovascular diseases. Curr Opin Mol Ther, 10, 611-621.

Hastings, C. L., Roche, E. T., Ruiz-Hernandez, E., Schenke-Layland, K., Walsh, C. J., \& Duffy, G. P. (2014). Drug and cell delivery for cardiac regeneration. Adv Drug Deliv Rev.

Hedger, M. P., \& de Kretser, D. M. (2013). The activins and their binding protein, follistatin-Diagnostic and therapeutic targets in inflammatory disease and fibrosis. Cytokine Growth Factor Rev, 24, 285-295.

Hill-Kapturczak, N., Jarmi, T., \& Agarwal, A. (2007). Growth factors and heme oxygenase-1: perspectives in physiology and pathophysiology. Antioxid Redox Signal, 9, 2197-2207.

Hinck, A. P. (2012). Structural studies of the TGF-betas and their receptors - insights into evolution of the TGF-beta superfamily. Febs Letters, 586, 1860-1870.

Ho, D. M., Yeo, C. Y., \& Whitman, M. (2010). The role and regulation of GDF11 in Smad2 activation during tailbud formation in the Xenopus embryo. Mech Dev, 127, 485-495.

Hsueh, Y. C., Wu, J. M., Yu, C. K., Wu, K. K., \& Hsieh, P. C. (2014). Prostaglandin E (2) promotes postinfarction cardiomyocyte replenishment by endogenous stem cells. EMBO Mol Med, 6, 496503.

Jabbour, A., Hayward, C. S., Keogh, A. M., Kotlyar, E., McCrohon, J. A., England, J. F., Amor, R., Liu, X., Li, X. Y., Zhou, M. D., Graham, R. M., \& Macdonald, P. S. (2011). Parenteral administration of recombinant human neuregulin-1 to patients with stable chronic heart failure produces favourable acute and chronic haemodynamic responses. Eur J Heart Fail, 13, 83-92.

Jeong, J. Y., Silver, M., Parnes, A., Nikiforow, S., Berliner, N., \& Vanasse, G. J. (2011). Resveratrol ameliorates TNFalpha-mediated suppression of erythropoiesis in human CD34 (+) cells via modulation of NF-kappaB signalling. Br J Haematol, 155, 93-101.

Jung, D. W., \& Williams, D. R. (2012). Reawakening atlas: chemical approaches to repair or replace dysfunctional musculature. ACS Chem Biol, 7, 1773-1790.

Katsimpardi, L., Litterman, N. K., Schein, P. A., Miller, C. M., Loffredo, F. S., Wojtkiewicz, G. R., Chen, J. W., Lee, R. T., Wagers, A. J., \& Rubin, L. L. (2014). Vascular and neurogenic rejuvenation of the aging mouse brain by young systemic factors. Science, 344, 630-634.

Kim, J., Wu, H. H., Lander, A. D., Lyons, K. M., Matzuk, M. M., \& Calof, A. L. (2005). GDF11 controls the timing of progenitor cell competence in developing retina. Science, 308, 1927-1930.

Kondas, K., Szlama, G., Nagy, A., Trexler, M., \& Patthy, L. (2011). Biological functions of the WAP domain-containing multidomain proteins WFIKKN1 and WFIKKN2. Biochem Soc Trans, 39, 1416-1420.

Kondas, K., Szlama, G., Trexler, M., \& Patthy, L. (2008). Both WFIKKN1 and WFIKKN2 have high affinity for growth and differentiation factors 8 and 11. J Biol Chem, 283, 23677-23684.

Korf-Klingebiel, M., Kempf, T., Sauer, T., Brinkmann, E., Fischer, P., Meyer, G. P., Ganser, A., Drexler, H., \& Wollert, K. C. (2008). Bone marrow cells are a rich source of growth factors and cytokines: implications for cell therapy trials after myocardial infarction. Eur Heart J, 29, 2851-2858. 
Langdon, J. M., Barkataki, S., Berger, A. E., Cheadle, C., Xue, Q. L., Sung, V., \& Roy, C. N. (2015). RAP011, an activin receptor ligand trap, increases hemoglobin concentration in hepcidin transgenic mice. Am J Hematol, 90, 8-14.

Lee, Y. S., \& Lee, S. J. (2013). Regulation of GDF-11 and myostatin activity by GASP-1 and GASP-2. Proc Natl Acad Sci U S A, 110, E3713-3722.

Leung, R., Proitsi, P., Simmons, A., Lunnon, K., Guntert, A., Kronenberg, D., Pritchard, M., Tsolaki, M., Mecocci, P., Kloszewska, I., Vellas, B., Soininen, H., Wahlund, L. O., \& Lovestone, S. (2013). Inflammatory proteins in plasma are associated with severity of Alzheimer's disease. PLoS One, 8, e64971.

Loffredo, F. S., Steinhauser, M. L., Jay, S. M., Gannon, J., Pancoast, J. R., Yalamanchi, P., Sinha, M., Dall'Osso, C., Khong, D., Shadrach, J. L., Miller, C. M., Singer, B. S., Stewart, A., Psychogios, N., Gerszten, R. E., Hartigan, A. J., Kim, M. J., Serwold, T., Wagers, A. J., \& Lee, R. T. (2013). Growth differentiation factor 11 is a circulating factor that reverses age-related cardiac hypertrophy. Cell, 153, 828-839.

Marinkovic, D., Zhang, X., Yalcin, S., Luciano, J. P., Brugnara, C., Huber, T., \& Ghaffari, S. (2007). Foxo3 is required for the regulation of oxidative stress in erythropoiesis. J Clin Invest, 117, 21332144.

Massague, J. (1998). TGF-beta signal transduction. Annu Rev Biochem, 67, 753-791.

McPherron, A. C. (2010). Metabolic Functions of Myostatin and Gdf11. Immunol Endocr Metab Agents Med Chem, 10, 217-231.

Pardali, E., \& Ten Dijke, P. (2012). TGFbeta signaling and cardiovascular diseases. Int J Biol Sci, 8, 195213.

Patel, K., \& Amthor, H. (2005). The function of Myostatin and strategies of Myostatin blockade-new hope for therapies aimed at promoting growth of skeletal muscle. Neuromuscul Disord, 15, 117-126.

Paulson, R. F. (2014). Targeting a new regulator of erythropoiesis to alleviate anemia. Nat Med, 20, 334-335.

Raje, N., \& Vallet, S. (2010). Sotatercept, a soluble activin receptor type 2A IgG-Fc fusion protein for the treatment of anemia and bone loss. Curr Opin Mol Ther, 12, 586-597.

Rochette, L., Cottin, Y., Zeller, M., \& Vergely, C. (2013). Carbon monoxide: mechanisms of action and potential clinical implications. Pharmacol Ther, 137, 133-152.

Rochette, L., Gudjoncik, A., Guenancia, C., Zeller, M., Cottin, Y., \& Vergely, C. (2014). The ironregulatory hormone hepcidin: A possible therapeutic target? Pharmacol Ther.

Rochette, L., Lorin, J., Zeller, M., Guilland, J. C., Lorgis, L., Cottin, Y., \& Vergely, C. (2013). Nitric oxide synthase inhibition and oxidative stress in cardiovascular diseases: possible therapeutic targets? Pharmacol Ther, 140, 239-257.

Ruckle, J., Jacobs, M., Kramer, W., Pearsall, A. E., Kumar, R., Underwood, K. W., Seehra, J., Yang, Y., Condon, C. H., \& Sherman, M. L. (2009). Single-dose, randomized, double-blind, placebocontrolled study of ACE-011 (ActRIIA-IgG1) in postmenopausal women. J Bone Miner Res, 24, 744-752.

Sanchis-Gomar, F., Garcia-Gimenez, J. L., Pareja-Galeano, H., Romagnoli, M., Perez-Quilis, C., \& Lippi, G. (2014). Erythropoietin and the heart: physiological effects and the therapeutic perspective. Int J Cardiol, 171, 116-125.

Sanganalmath, S. K., \& Bolli, R. (2013). Cell therapy for heart failure: a comprehensive overview of experimental and clinical studies, current challenges, and future directions. Circulation Research, 113, 810-834.

Segers, V. F., \& Lee, R. T. (2010). Protein therapeutics for cardiac regeneration after myocardial infarction. J Cardiovasc Transl Res, 3, 469-477.

Sherman, M. L., Borgstein, N. G., Mook, L., Wilson, D., Yang, Y., Chen, N., Kumar, R., Kim, K., \& Laadem, A. (2013). Multiple-dose, safety, pharmacokinetic, and pharmacodynamic study of sotatercept (ActRIIA-IgG1), a novel erythropoietic agent, in healthy postmenopausal women. J Clin Pharmacol, 53, 1121-1130. 
Shi, Y., \& Liu, J. P. (2011). Gdf11 facilitates temporal progression of neurogenesis in the developing spinal cord. J Neurosci, 31, 883-893.

Sinha, M., Jang, Y. C., Oh, J., Khong, D., Wu, E. Y., Manohar, R., Miller, C., Regalado, S. G., Loffredo, F. S., Pancoast, J. R., Hirshman, M. F., Lebowitz, J., Shadrach, J. L., Cerletti, M., Kim, M. J., Serwold, T., Goodyear, L. J., Rosner, B., Lee, R. T., \& Wagers, A. J. (2014). Restoring systemic GDF11 levels reverses age-related dysfunction in mouse skeletal muscle. Science, 344, 649652.

Souza, T. A., Chen, X., Guo, Y., Sava, P., Zhang, J., Hill, J. J., Yaworsky, P. J., \& Qiu, Y. (2008). Proteomic identification and functional validation of activins and bone morphogenetic protein 11 as candidate novel muscle mass regulators. Mol Endocrinol, 22, 2689-2702.

Suragani, R. N., Cadena, S. M., Cawley, S. M., Sako, D., Mitchell, D., Li, R., Davies, M. V., Alexander, M. J., Devine, M., Loveday, K. S., Underwood, K. W., Grinberg, A. V., Quisel, J. D., Chopra, R., Pearsall, R. S., Seehra, J., \& Kumar, R. (2014). Transforming growth factor-beta superfamily ligand trap ACE-536 corrects anemia by promoting late-stage erythropoiesis. Nat Med, 20, 408-414.

Tanno, T., Bhanu, N. V., Oneal, P. A., Goh, S. H., Staker, P., Lee, Y. T., Moroney, J. W., Reed, C. H., Luban, N. L., Wang, R. H., Eling, T. E., Childs, R., Ganz, T., Leitman, S. F., Fucharoen, S., \& Miller, J. L. (2007). High levels of GDF15 in thalassemia suppress expression of the iron regulatory protein hepcidin. Nat Med, 13, 1096-1101.

Tesoriere, L., D'Arpa, D., Butera, D., Allegra, M., Renda, D., Maggio, A., Bongiorno, A., \& Livrea, M. A. (2001). Oral supplements of vitamin E improve measures of oxidative stress in plasma and reduce oxidative damage to $L D L$ and erythrocytes in beta-thalassemia intermedia patients. Free Radic Res, 34, 529-540.

Tsuchida, K., Nakatani, M., Uezumi, A., Murakami, T., \& Cui, X. (2008). Signal transduction pathway through activin receptors as a therapeutic target of musculoskeletal diseases and cancer. Endocr J, 55, 11-21.

Tuszynski, G. P., Rothman, V. L., Zheng, X., Gutu, M., Zhang, X., \& Chang, F. (2011). G-protein coupled receptor-associated sorting protein 1 (GASP-1), a potential biomarker in breast cancer. Exp Mol Pathol, 91, 608-613.

Villeda, S. A., Plambeck, K. E., Middeldorp, J., Castellano, J. M., Mosher, K. I., Luo, J., Smith, L. K., Bieri, G., Lin, K., Berdnik, D., Wabl, R., Udeochu, J., Wheatley, E. G., Zou, B., Simmons, D. A., Xie, X. S., Longo, F. M., \& Wyss-Coray, T. (2014). Young blood reverses age-related impairments in cognitive function and synaptic plasticity in mice. Nat Med, 20, 659-663.

Wang, L., Di, L., \& Noguchi, C. T. (2014). Erythropoietin, a novel versatile player regulating energy metabolism beyond the erythroid system. Int J Biol Sci, 10, 921-939.

Wang, L., Karpac, J., \& Jasper, H. (2014). Promoting longevity by maintaining metabolic and proliferative homeostasis. J Exp Biol, 217, 109-118. 
Figure 1

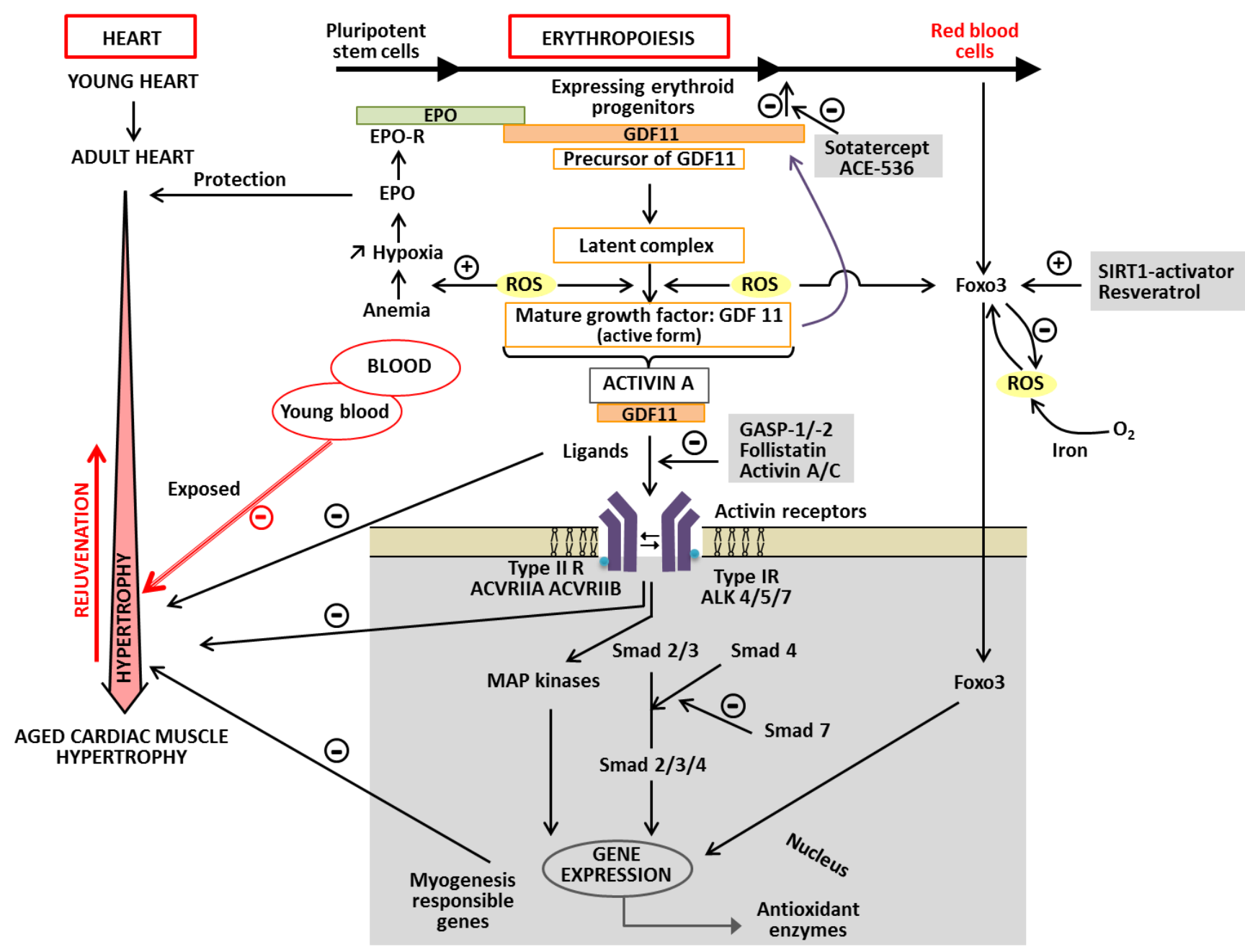


Figure 2

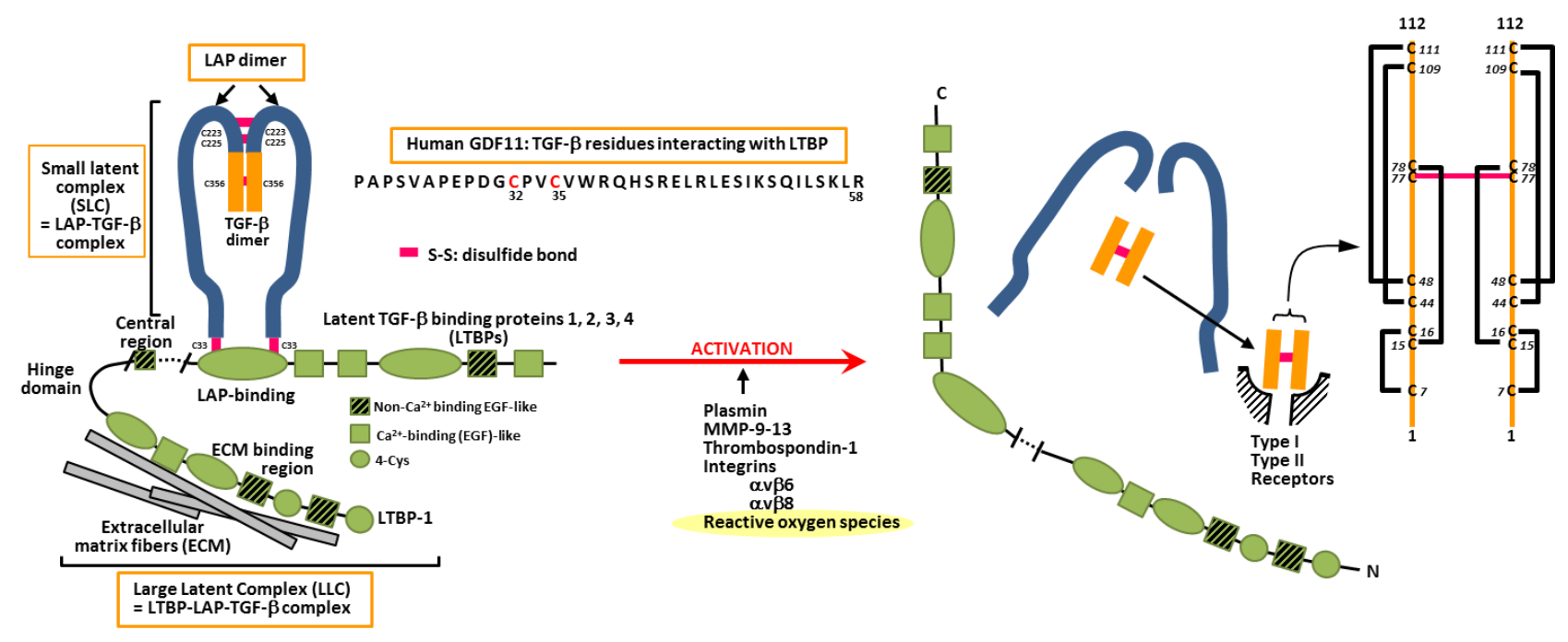


Figure 3

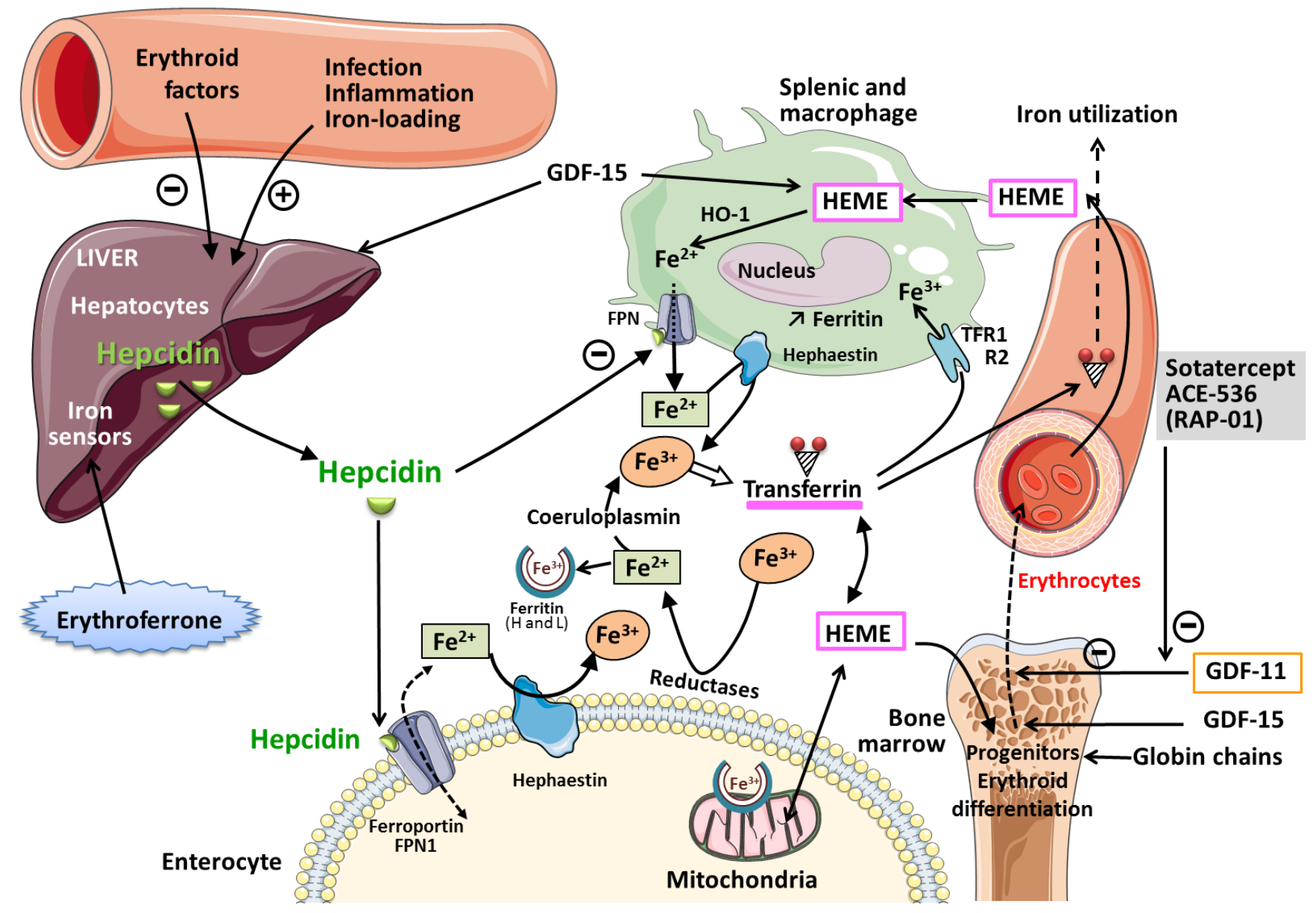


